December 12, 2002

\title{
IMPROVING LINK-STATE ROUTING - BY USING ESTIMATED FUTURE LINK DELAYS (REVISED) $^{+* \times}$
}

\author{
Hyeonsang Eom \\ hseom@cs.umd.edu \\ Institute for Advanced Computer Studies \\ Computer Science Department \\ University of Maryland \\ College Park, 20742 \\ CS-TR-4297R \\ UMIACS-TR-2001-75R
}

December 12, 2002

\begin{abstract}
In link-state routing, routes are determined based on estimates of the current delays on the links. Ideally, a data packet should be routed based on the delays it will encounter at each link of the path at the time the packet gets to the link. To address this issue, we have developed a new approach that improves link-state routing by estimating and using the future link delays encountered by data packets. In link-state routing, link-delay estimates are periodically flooded throughout the network. This flooding of link-delay estimates is done without considering the relevance of these estimates to routing quality. Our approach also improves link-state routing by broadcasting these estimates only to the extent that they are relevant. Simulation studies
\end{abstract}

\footnotetext{
${ }^{+}$This work is supported partly by DARPA/Rome Labs, Department of the Air Force, under contract F306020020578 to the Department of Computer Science at the University of Maryland. The views, opinions, and/or findings contained in this report are those of the author(s) and should not be interpreted as representing the official policies, either expressed or implied, of the Department of Air Force, DARPA, DOD, or the U.S. Government.

* This work was supported in part by the Maryland Information and Network Dynamics (MIND) Laboratory, its Founding Partner Fujitsu Laboratories of America, and by the Department of Defense through a University of Maryland Institute for Advanced Computer Studies (UMIACS) contract.

${ }^{x}$ This report is a revised version of the report titled Information Dynamics applied to link-state routing.
} 
suggest that our approach can lead to significant reductions in routing traffic with noticeable improvements of routing quality in high-load conditions.

\section{Introduction}

In a packet switched network a collection of nodes, consisting of computing machines, is connected using communication links capable of transferring information in the form of packets, from one node to another. When a direct link does not exist from the source node and the destination node, but a path via one or more intermediate nodes exists, the packets from the source node follow this path, such that each intermediate node carries out a storeand-forward operation until the packets are delivered to the destination node. The path is determined through "routing" techniques. Clearly routing is an operation which has to reply on the global state of the network. Several techniques have been used for collecting and using global information for routing. One such technique is link-state routing in which each node collects information on the state of its outgoing links, usually in the form of the waiting time, and shares it with all other nodes in the network. Based on the link-state information collected, a node may determine the "best" path from it to any other node of the network.

In link-state routing, we refer to the global link-state information that each node maintains and uses for routing, as a view. The view is essentially a graph with vertices corresponding to the network nodes, edges corresponding to network links, and for each link, a cost representing an estimate of the current delay on the link. Based on its view, each node determines least-cost paths to all other nodes as the best paths. The end-to-end cost function is the sum of the costs at all the links in the route.

Each node makes (periodic and/or event-driven) measurements of the current delay for each of its outgoing links. It periodically constructs an estimate for the current delay on the link from these measurements as link-cost estimates, and sends these link-delay estimates to all other nodes in the network. When broadcasting, a node sends link-delay estimates for its outgoing links to its neighbors, and each of the neighbors in turn sends these estimates to its neighbors, and this continues until all nodes receive the estimates. This broadcast technique is referred to as flooding [Peterson, 1996; Rosen, 1980]. When each node constructs a link-delay estimate for a local link (i.e., a link going from it) or receives an estimate for a remote link, it updates its view (view update). Each node periodically uses its current information about the 
network to compute least-cost paths to all other nodes (periodic route update) by using the standard shortest-path algorithm [Dijkstra, 1959]. These least-cost paths are used by the node to route data packets, i.e. when the node receives a data packet, it forwards the packet to the neighbor that is the next node in the least-cost path to the destination node of the packet.

Consider a node with a link $l$ outgoing from it. Let $x$ denote the delay on the link and $t$ represent time. Assume that this node has made delay measurements, $x_{-n}, x_{-(n-1)}, \ldots, x_{-1}$ on this link at time $t_{-n}, t_{-(n-1)}, \ldots, t_{-1}$, respectively, before the current time $t_{0}$, where $n$ is the total number of measurements and time $t_{i}$ precedes $t_{j}$ if $i<j$. Say the node makes an estimate of the current link delay using these previous delay measurements. Let $\hat{x}_{0}\left(x_{-n}, x_{-(n-1)}, \ldots, x_{-1}\right)$ denote this delay estimate. In link-state routing, $\hat{x}_{0}\left(x_{-n}, x_{-(n-1)}, \ldots, x_{-1}\right)$ is periodically computed and flooded to all other nodes so that each node can use this estimate as the cost of link $l$ when computing least-cost paths to all other nodes.

In link state routing, there is a key issue to consider: each node creates a delay estimate $\hat{x}$ for an outgoing link and sends it to all nodes. This estimate is based on measurements made in the past and its value to a receiving node may decrease over time, to the point that the new estimate may not lead to any changes in the view of a node receiving it. In this report, we examine this issue by reflecting the changes in the estimates over time and take such changes into account for not only the routing decisions but also the flooding decisions.

\subsection{Link State}

Many variations of the standard link-state-routing approach described above have been proposed, where link-state information in forms other than that of link delays is used in routing (this means that link costs do not represent delays). This is primarily because the ways that link delays have been used can lead to inferior routing performance, say due to routing instabilities. For example, routing performance can be degraded because of routing oscillations, where given two links connecting two regions of a network, the preferred route from one region to the other for most or all inter-region traffic is constantly switched because most or all traffic shifts at the same time due to all nodes' simultaneous adjustment of their routes with the same reported delay values for the links [Khanna, 1989]. 
Historically, the original ARPANET used queue length as the link-cost metric. However, this metric was not effective because it did not take either link bandwidth or the latency into account. The original scheme was revised to consider the bandwidth and latency by using delay averages as link costs. However, this revised scheme caused routing instabilities under heavy load, resulting in routing oscillations. For stable routing, the ARPANET metric was again revised to a hop-normalized-utilization function [Khanna, 1989]. This function computes a link cost by normalizing an estimate of the link utilization via a linear transformation after making this estimate with measured delay values. (The idea behind this computation is hopnormalization, which means normalization of the utilization in terms of hops so that the resulting cost is relative to that of alternative links. To illustrate how routing is done based on this idea, consider the case where 61 is reported as the cost of a link $l_{1}$ and 20 is reported as that of an alternative link $l_{2}$. In this case, $l_{2}$ with additional 2 hops is used in routing before $l_{1}$.) This new approach smoothed the temporal variation of the metric by using link utilization rather than link delay and using a movement (metric-change) limit, and compressed the dynamic range of the metric by limiting the cost-value range and considering the link type. However, the benefit of using this kind of technique to damp out sudden changes or routing oscillations depends on the network and/or traffic pattern. Recently, there was an attempt to tune the parameters in the link-cost function via online simulation [Kaur, 2000]. Also, congestion-based metrics were proposed [Glazor, 1990]. However, these metrics have been unpopular because the use of the metrics may lead to routing instabilities [Kaur, 2000].

In some cases, link costs are not recalculated dynamically and constant link costs are used most of the times. In these cases, the computation and broadcast of link costs for route updates are not periodic. For example, link costs are set statically by a network administrator, and these costs are changed only in case of link failure. This static approach may be effective in some small-scale networks in which there are few or no alternative paths. However, the approach may not be suitable for most networks in which there are many alternate paths, because different alternate paths may become more or less desirable as link delays change over time.

\subsection{Approach}

In link-state routing, each node makes link-delay estimates based solely on information provided in the past, and uses these estimates in routing. Ideally, a data packet should be 
routed based on the delays it will encounter at each link of the path at the time the packet gets to the link. That is, for each link along a potential route, the node doing the routing needs an estimate of the link delay at the (future) time when the data packet would arrive at the link. We refer to this future delay as encountered delay. To address the issue that routing should be done based on encountered delay, we have developed a new approach to consider encountered delay in routing by estimating this delay via a projection technique. This technique is described in Section 3. Before presenting our approach, Section 2 provides a formal description of the problem of estimating encountered delay and using this delay to determine least-cost paths in routing.

Also, in link-state routing, each node floods its link-cost estimates without regard to whether the estimates are necessary for determining least-cost paths. This could result in a significant amount of unnecessary routing traffic. In our approach, each node disseminates link-cost information only when necessary for estimating encountered delay. We have found that our approach leads to reductions in routing traffic as well as improvements in data performance (e.g. delay, throughput). Section 3 also presents this selective-broadcast technique. Our experiments and the results are presented in Section 4. In this section, we describe the network configuration and scenarios for our simulation studies, and present the comparison results obtained from these studies. Section 5 briefly surveys major related works. Finally, Section 6 concludes our work and summarizes our future work. 


\section{Problem Formulation}

For a link, we treat the delay $x$ at time $t$ as a stationary stochastic process $\{x(t)\}$. Thus, the mean and variance of $x(t)$ are constant (independent of time $t$ ). Let $m$ and $\sigma^{2}$ denote the mean and variance, respectively. Also, the autocorrelation function $E[\{x(t)-m\}\{x(t+\tau)-m\}] / \sigma^{2}$ depends only on the lag $\tau$ and not on time $t$. Let $\rho(\tau)$ denote this autocorrelation function.

Consider the instantaneous conditional mean and variance, respectively, of the delay given a measurement $x_{0}$ at time $t_{0}$ :

$$
\begin{gathered}
E\left\{x(t) \mid x\left(t_{0}\right)=x_{0}\right\}, \text { where } t_{0}<t \\
\operatorname{Var}\left\{x(t) \mid x\left(t_{0}\right)=x_{0}\right\}, \text { where } t_{0}<t
\end{gathered}
$$

If no other measurement is available, we expect the instantaneous conditional mean to change from $x_{0}$ towards $m$ over time. Similarly, we expect that the instantaneous conditional variance to change from zero to $\sigma^{2}$ over time. When the measurement is made, the conditional variance is zero because the true value of $x$ is observed at that time.

For the determination of least encountered-delay paths and the selective broadcast of linkdelay information, we require estimates for the functions $E\left\{x(t) \mid x\left(t_{0}\right)=x_{0}\right\}$ and $\operatorname{Var}\left\{x(t) \mid x\left(t_{0}\right)=x_{0}\right\}$. Based on the steady-state behavior explained above, we need techniques for estimating these function values. The routing problem that we address is how to use these estimates in route determination and selective broadcast. 


\section{Approach}

We assume that the conditional mean decays exponentially over time to its steady-state value. Based on this assumption, we use

$$
\hat{m}\left(x_{0}, t_{0}, t\right)=x_{0}+\left(m-x_{0}\right)\left(1-e^{-\alpha\left(t-t_{0}\right)}\right)\left(t \geq t_{0}\right)
$$

as an estimate of $E\left\{x(t) \mid x\left(t_{0}\right)=x_{0}\right\}$, where $\alpha$ is a non-negative constant. This is illustrated in Figure 1. Similarly, we use an exponential-decaying estimate $\hat{\sigma}^{2}\left(x_{0}, t_{0}, t\right)$ for $\operatorname{Var}\left\{x(t) \mid x\left(t_{0}\right)=x_{0}\right\}$, as illustrated in Figure 2.

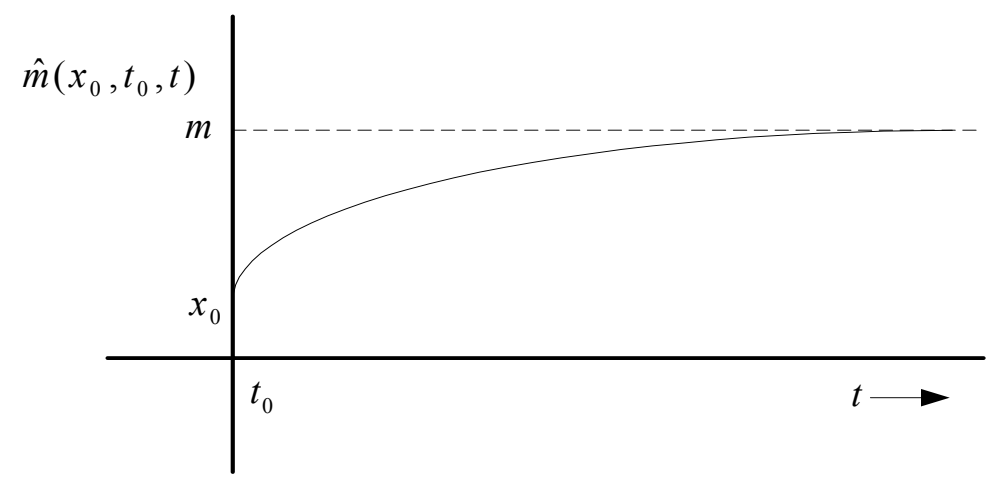

Figure 1 Evolution of the instantaneous conditional delay-mean estimate

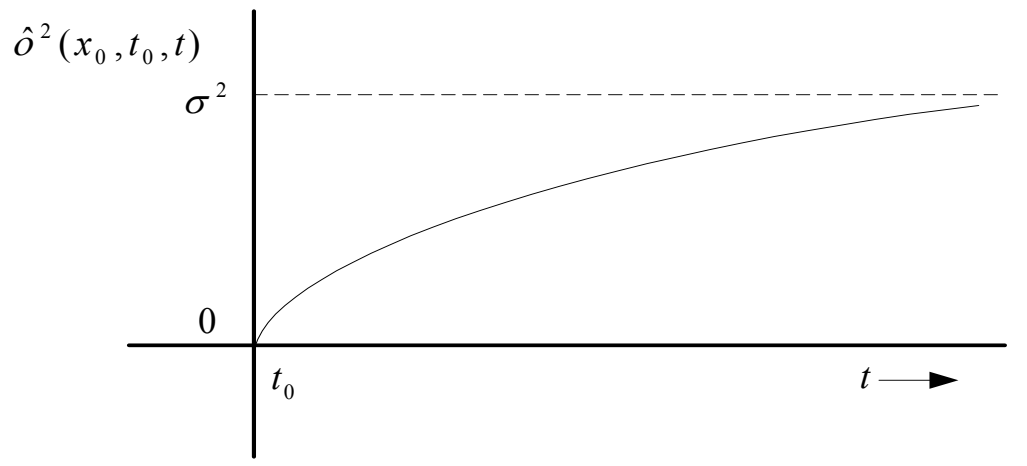

Figure 2 Evolution of the instantaneous conditional delay-variance estimate 
Theorem If $x(t)$ is a stochastic process and has the following additive form for a constant $m$ and a positive constant $\Delta t$ :

$$
x(t+\Delta t)-m=\beta\{x(t)-m\}+(1-\beta) v(t),
$$

then

(i) $E\{x(t)\}=m, \operatorname{Var}\{x(t)\}$ is a constant (denoted by $\sigma^{2}$ ), and the autocorrelation function

$$
\rho\left(t, t_{0}\right)=E\left[\left\{x\left(t_{0}\right)-m\right\}\{x(t)-m\}\right] / \sigma^{2}
$$

$\left(t=t_{0}+k(\Delta t)\right.$ for a non-negative integer $k$ ) decreases exponentially as $t-t_{0}$ (or $k$ ) increases (depending only on the lag), and

(ii) $E\left\{x(t) \mid x\left(t_{0}\right)=x_{0}\right\}=x_{0}+\left(m-x_{0}\right)\left\{1-\rho\left(t, t_{0}\right)\right\}$,

where $\beta$ is a constant such that $0<\beta<1$, and $v(t)$ is an independent white-noise process such that $E\{v(t)\}=0$ and $\operatorname{Var}\{v(t)\}=E\left\{v^{2}(t)\right\}=\sigma_{v}^{2}$ (positive constant) $\square$ (proof in Appendix A on pages 23 to 26).

Note that this theorem is valid for arbitrary $t_{0}$ and $t \geq t_{0}$ if there is a non-negative integer $k$ such that $t=t_{0}+k(\Delta t)$, where $\Delta t$ satisfies the additive model form. We can choose such $k$ and $\Delta t$. Thus, we can apply the theorem with respective to arbitrary $t_{0}$ and $t \geq t_{0}$.

Part (i) of the theorem implies that if $x(t)$ has the additive form, then $x(t)$ is stationary and its autocorrelation function decreases exponentially in time. When we examined a sample autocorrelation function for a set of Internet round-trip delay measurements made using the NetDyn tool [Sanghi, 1993], we found that the function decays exponentially in the short term. The additive form is a form that we work with. Based on Part (ii) of the theorem, we make the assumption stated at the beginning of this section, that the conditional mean decays exponentially over time. Refer to Appendix B (pages 27 and 28) for the additional theorem that explains our motivation of using the exponential-decaying conditional delay-variance estimate. 
Figure 3 shows a sample of Internet round-trip delay measurements, and Figure 4 shows a sample autocorrelation function computed for this sample. The least-square estimates of $\alpha$ for different sets of Internet round-trip delay measurements range from $58 \mathrm{sec}^{-1}$ to $73 \mathrm{sec}^{-1}$. Table 1 shows these values.

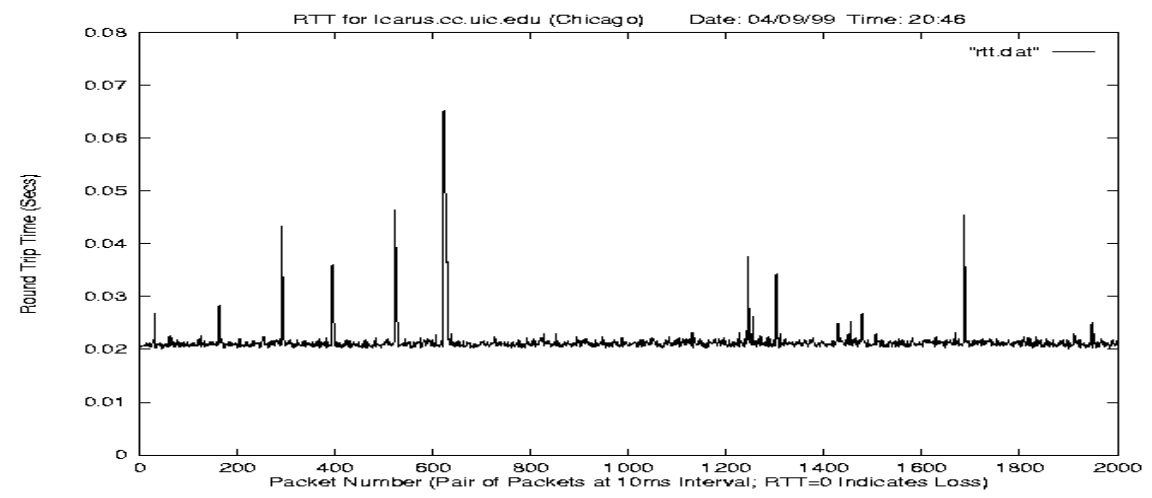

Figure 3 Round-trip times measured at $10 \mathrm{~ms}$ intervals between the University of Maryland at College Park and the University of Illinois at Chicago for 10 seconds from $20: 46$ on $04 / 09 / 99$

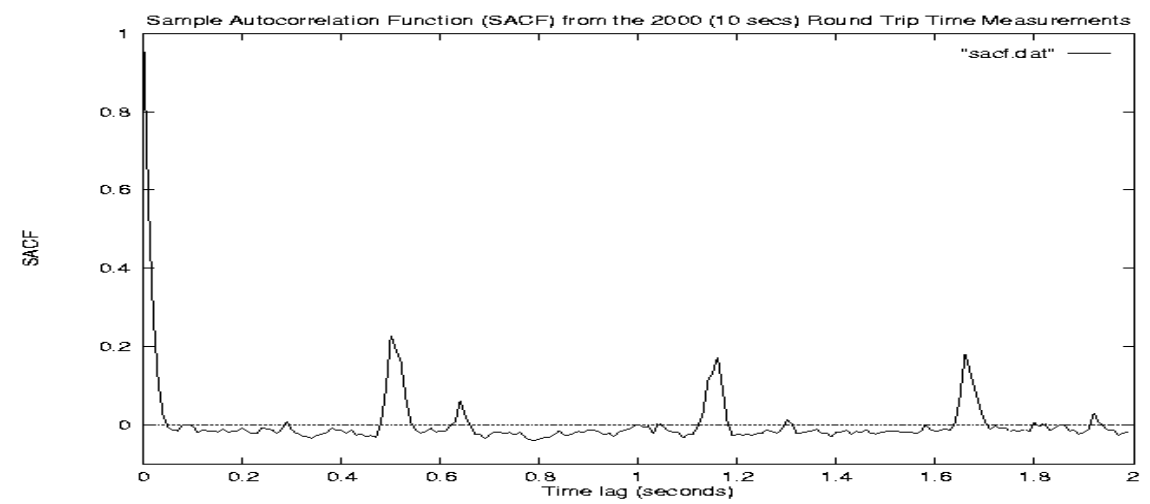

Figure 4 Sample autocorrelation function

Table $1 \alpha$ values estimated with round-trip delay measurements made at $10 \mathrm{~ms}$ intervals between Maryland and Illinois for different start-times and durations on $04 / 09 / 99$ (via sample autocorrelation functions)

\begin{tabular}{c|ccc}
\hline \multirow{2}{*}{ Start-Time } & \multicolumn{3}{|c}{ Duration (Seconds) } \\
\cline { 2 - 4 } & 10 & 20 & 30 \\
\hline $20: 46$ & 67 & 71 & 58 \\
$20: 51$ & 73 & 66 & 69 \\
\hline
\end{tabular}


Given these exponential-decaying estimation functions, a node computes the encountered delay of a packet on a path as follows. Let the path have links $l_{1}, l_{2}, \ldots, l_{n}$, and let the node send the packet into the path at time $t_{0}$. Let $\hat{m}^{l_{i}}(t)$ be the function estimating the encountered delay on link $l_{i}$ at time $t$. The estimated encountered delay for the packet on link $l_{1}$ is $\hat{m}^{l_{1}}\left(t_{0}\right)$. The estimated encountered delay for the packet on link $l_{2}$ is $\hat{m}^{l_{2}}\left(t_{0}+\hat{m}^{l_{1}}\left(t_{0}\right)\right)$, and so on. So the estimated encountered delay for the packet on the path is given by:

$$
\hat{m}^{l_{1}}\left(t_{0}\right)+\hat{m}^{l_{2}}\left(t_{0}+\hat{m}^{l_{1}}\left(t_{0}\right)\right)+\hat{m}^{l_{3}}\left(t_{0}+\hat{m}^{l_{1}}\left(t_{0}\right)+\hat{m}^{l_{2}}\left(t_{0}+\hat{m}^{l_{1}}\left(t_{0}\right)\right)\right)+\ldots+\hat{m}^{l_{n}}(\ldots)
$$

Computing path costs in this way, the node would route the packet on the path with the least encountered-delay estimate. Each node determines the least-cost paths using the standard shortest-path algorithm as in link-state routing. Thus, routing is carried out as in standard linkstate routing except for the path-cost computation. To do this computation, each node maintains a view as in link-state routing except that a measured delay and measurement time are kept for each link. The node updates its view of a local link whenever a data packet is sent on that link. The node updates its view of a remote link whenever it receives a measurement update for the link. View updates are not periodic. Note that in our approach, link costs are not considered as part of the link views in contrast to link-state routing.

At each view update for a link, each node updates the measured delay and measurement time that it maintains for the link. Each node broadcasts the updated delay information to its neighbors only if the estimated encountered delay on the corresponding link at that time is significantly different from the steady-state mean. We assume that every node knows the steady-state value of delay on each link. In our approach, the estimated encountered delay becomes close to the corresponding steady-state value over time. Hence, no propagation of updated delay information is required beyond some point. If a node does not receive any measurement update for a link, it uses the steady-state value.

Each node maintains a routing table that indicates the next hop for each destination as in linkstate routing. In link-state routing, with its view for all links, each node periodically updates its routing table by computing the least-cost paths to all the other nodes. However, in our approach, each node computes link costs and updates routes just before it decides which of its outgoing links to send the packet onto when it receives a data packet. We refer to this update 
technique as the "just-in-time route-update" method. This method allows each node to determine the current least-cost paths using the encountered delay estimated with the most recent delay information for each link. Note that the periodic-update scheme used in link-state routing is not suitable for our approach. The reason is that if the periodic-update scheme were used, routes determined using our approach at each route-update time would be used without any change until the next route-update time. The problem with this is that temporally-changing estimated encountered delays cannot be used for routing of individual data packets. If linkdelay estimates made in the case of using the periodic scheme are close to the steady-state values, the result of using the periodic scheme in our routing approach could be comparable to that of using link-state routing. However, these link-delay estimates can be different from the steady-state values. For example, consider the case where periodic route updates occur right after link delays that are different from the steady-state values are measured. If these measured values are used to make link-delay estimates at these route updates, these estimates are close or equal to the measured values that are different from the steady-state values. Thus, the result of using the periodic scheme can be negative.

We refer to our routing approach based on estimates of encountered delays as InfoDyn routing. Table 2 summarizes the commonalities and differences between InfoDyn and the standard link state routing approaches. In both approaches, each node measures the delay of a data packet on each local link by time-stamping its arrival at the node and its departure from the link queue, and adding this time difference to the sum of the packet transmission time (the packet size divided by the link bandwidth) and link-propagation delay. We refer to a particular node as a measurement node, and a node that uses the delay measurements made by this measurement node for routing as a routing node. In this table, we focus on the measurement node, local link, and routing node. 
Table 2 Comparison of InfoDyn routing with standard link-state routing

\begin{tabular}{|c|c|c|c|}
\hline \multicolumn{2}{|l|}{ Aspect } & InfoDyn Routing & Standard Link-State Routing \\
\hline \multirow{4}{*}{ Link-View Construction } & What & $\begin{array}{l}\text { Measurement and } \\
\text { Measurement Time }\end{array}$ & $\begin{array}{l}\text { Exponential Delay Average } \\
\text { (Link Cost) }\end{array}$ \\
\hline & Who & Measurement Node & Measurement Node \\
\hline & How & Measurement & $\begin{array}{l}\text { Exponential Averaging after } \\
\text { Measurement }\end{array}$ \\
\hline & When & $\begin{array}{l}\text { Measurement Time } \\
\text { (for View Update; When } \\
\text { Each Data Packet Is Sent } \\
\text { Out) }\end{array}$ & $\begin{array}{l}\text { Periodically } \\
\text { (for View Update) }\end{array}$ \\
\hline \multirow{2}{*}{ Link-View Transmission } & How & Conditional Broadcast & Unconditional Flooding \\
\hline & When & $\begin{array}{l}\text { Measurement Time } \\
\text { (for View Update) }\end{array}$ & $\begin{array}{l}\text { Periodically } \\
\text { (for View Update) }\end{array}$ \\
\hline \multirow[b]{2}{*}{ View Update } & Who & Routing Node & Routing Node \\
\hline & When & $\begin{array}{l}\text { When Each View Update Is } \\
\text { Received }\end{array}$ & $\begin{array}{l}\text { When Each View Update Is } \\
\text { Received }\end{array}$ \\
\hline \multirow{4}{*}{ Link-Cost Computation } & What & $\begin{array}{l}\text { Encountered-Delay } \\
\text { Estimate }\end{array}$ & Exponential Delay Average \\
\hline & Who & Routing Node & Measurement Node \\
\hline & How & $\begin{array}{l}\text { Exponential Model } \\
\text { (with View) }\end{array}$ & $\begin{array}{l}\text { Exponential Averaging } \\
\text { (with Measurement) }\end{array}$ \\
\hline & When & Route Update & $\begin{array}{l}\text { Periodically } \\
\text { (for View Update and } \\
\text { Subsequent Route Update) }\end{array}$ \\
\hline \multirow{4}{*}{$\begin{array}{l}\text { Route Update } \\
\text { (Using Link Costs) }\end{array}$} & What & $\begin{array}{l}\text { Least Encountered-Delay- } \\
\text { Estimate Paths }\end{array}$ & $\begin{array}{l}\text { Least Current-Delay- } \\
\text { Estimate Paths }\end{array}$ \\
\hline & Who & Routing Node & Routing Node \\
\hline & How & $\begin{array}{l}\text { Standard Shortest-Path } \\
\text { Algorithm }\end{array}$ & $\begin{array}{l}\text { Standard Shortest-Path } \\
\text { Algorithm }\end{array}$ \\
\hline & When & Arrival of a Data Packet & $\begin{array}{l}\text { Periodically } \\
\text { (Just after View Update) }\end{array}$ \\
\hline
\end{tabular}




\section{Simulation}

To show the overall applicability of this approach to link-state routing, we compared via simulation a routing scheme using our approach with SPF (Shortest Path First), link-state routing technique. For simulation studies, we used MaRS, the Maryland Routing Simulator [Alaettinoglu, 1994; Shankar, 1992]. We tried SPF with two kinds of link-cost functions, a delay cost function and a hop-normalized-utilization function [Khanna, 1989].

\subsection{Network configuration and scenarios}

We conducted studies for the NSFNET-T1-backbone topology. In this configuration, there are 14 nodes connected via 21 links. Each link represents two one-way channels. Each node can process a data packet of 544 bytes in $1 \mathrm{~ms}$, and each link channel has $183 \mathrm{~KB} / \mathrm{s}(1.4$ Mbps) bandwidth. We assume that there is no propagation delay for each link because we found that simulation results are not sensitive to the setting of propagation delay.

In this network, a workload is generated by FTP source and sink pairs. These sources and sinks are connected to nodes. FTP is regulated by a flow-control mechanism and an acknowledgement mechanism with retransmission. The flow-control mechanism is a static window-based scheme implemented in MaRS. This scheme consists of two windows: produce and send windows. We set the produce-window size to infinity, and the send-window size to eight. Also, we use 120 seconds as the total simulated time.

There are two kinds of FTP flows: regular and on-off flows. In each regular flow, the source starts transmitting packets at time 0 , and sends as many packets as possible with an interpacket production delay of $1 \mathrm{~ms}$. For each on-off flow, there are alternating constant-length on and off intervals. Each on-off flow starts at a different time (from 0 to 24 seconds), and has a different length (from 20 to 120 seconds). Also, a certain number of packets are produced at once at the beginning of on intervals while no packets are produced during off intervals. The number of packets for each on interval is determined so that the packets of that number would be successively transmitted during the on interval without any flow-control mechanism and without any other flow. Specifically, the number is the length of an on interval divided by the transmission time of a data packet, where the transmission time is the packet size divided by the link bandwidth. 
We initially consider five scenarios in this network configuration: N0 - N4. The level of queuing delay of these scenarios is high: in the best cases (lowest-average-delay cases after cost-function-parameter tuning) of using SPF with 1 second route-update intervals, the average queuing-delay portions of the average round-trip delay per packet are around $94 \%$. Also, the utilizations (the average fractions of the time when packet queue size $>1$ ) are around 0.73. There are 121 FTP flows in Scenario N0 to N3, and 131 flows in Scenario N4. Table 3 shows the differences between the scenarios. In particular, Scenario N4 has two hot spots (each of which receives packets from every other node).

Table 3 Differences in the FTP-flow characteristics between scenarios

\begin{tabular}{c|ccc}
\hline Scenario & $\begin{array}{c}\text { Number } \\
\text { of Regular Flows }\end{array}$ & $\begin{array}{c}\text { Number } \\
\text { of On-Off Flows }\end{array}$ & $\begin{array}{c}\text { Length of On-Off } \\
\text { Intervals (Seconds) }\end{array}$ \\
\hline N0 & 60 & 61 & 5 \\
N1 & 60 & 61 & 10 \\
N2 & 60 & 61 & 15 \\
N3 & 0 & 121 & 5 \\
N4 & 55 & 76 & 5 \\
\hline
\end{tabular}

\subsection{Results}

For the InfoDyn scheme, we used an exponential-change-rate $(\alpha)$ value and a threshold value for the selective broadcast of routing packets, during each simulation run for each scenario. We tried seven threshold values. Also, we tried eight $\alpha$ values across the full value range in each of these different-threshold-value cases. As the steady-state value of each link in each simulation run using the InfoDyn scheme, we used the sample delay mean of the corresponding link computed in a simulation run using SPF with 1 second route-update intervals for the same scenario.

The use of the InfoDyn scheme without any routing-packet broadcast (thereby with only locallink view update) is called the InfoDyn Short-Term Steady-State (STSS) case. Hereafter, "best" means leading to the lowest average round-trip delay per packet.

\subsubsection{InfoDyn Short-Term Steady-State (STSS) case}

In each scenario, we compared the InfoDyn STSS case of using the best $\alpha$ of the exponential model with the best cases of using SPF with 1, 10, and 30 second route-update intervals - we obtained the best result of using SPF for each combination of a route-update-interval length 
and a scenario by tuning several cost-function parameters. In this comparison, the InfoDyn case results in a 3 to $8 \%$ reduction in the Average (Avg) Round-Trip (RT) delay per packet and a 4 to $22 \%$ reduction in the standard deviation (STD) in all scenarios. Figure 5 shows these reductions. Note that there are no routing packets sent out in this InfoDyn case while $75,642,7,602$, and 2,562 routing packets are sent out with 1, 10, and 30 second route-update intervals, respectively, in the SPF cases. These results imply that when every node knows the "long-term" steady-state delay-mean values of all links and uses our routing approach, flooding requirements can be significantly reduced (as in the InfoDyn STSS case) with noticeable reductions in the average delay and the variance, compared with the standard link-state routing approach where each node periodically broadcasts "short-term" steady-state values (exponential averages) for link delays.

\section{Reductions in the Avg RT Delay of InfoDyn STSS wrt SPF w/ Different Route-Update Intervals}

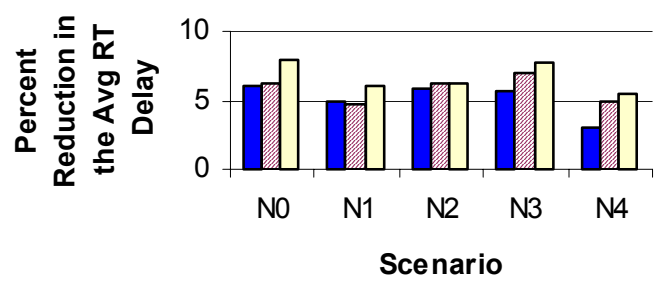

$1 \mathrm{sec}$ interval $\square 10 \mathrm{sec}$ interval $\square 30 \mathrm{sec}$ interval

\section{Reductions in the Avg-RT-Delay STD of InfoDyn STSS wrt SPF w/ Different Route-Update Intervals}

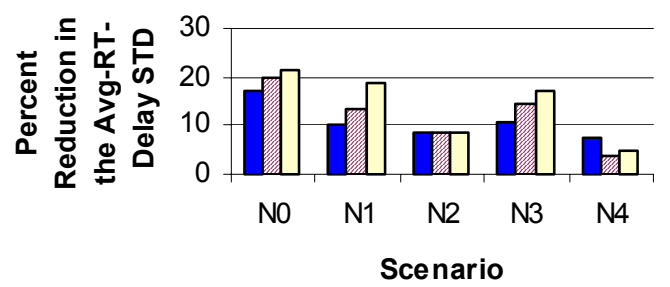

$1 \mathrm{sec}$ interval $\square 10 \mathrm{sec}$ interval $\square 30 \mathrm{sec}$ interval

Figure 5 Reductions in the average round-trip delay and STD of InfoDyn STSS

\subsubsection{Impact of routing-packet broadcast}

For each scenario with a fixed $\alpha$ value, the average delay and STD are almost the same across simulation runs with different threshold values, except for those runs in which a very large number of routing packets are broadcast. For example, Figure 6 shows the impact of varying the threshold value in Scenario N3 (the all-on-off case) when the best $\alpha$ is used. There are three charts. The left-most and middle charts indicate the changes in the average delay and STD, respectively, depending on the threshold value used. The right-most chart shows the numbers of routing packets used for different threshold values. The smaller the threshold value, the more routing packets are sent out. When about 40,000 routing packets are used (the $100 \mathrm{~ms}$ threshold-value case), there are $0.53 \mathrm{~ms}$ and $0.66 \mathrm{~ms}$ increases in the average delay and 
STD, respectively, compared with $152.03 \mathrm{~ms}$ average delay and $92.24 \mathrm{~ms}$ STD of the best case (the $130 \mathrm{~ms}$ threshold-value case). Similar impacts of routing-packet broadcast are observed for the other scenarios. Appendix C (Figure A1 on pages 29 and 30) shows the same three charts in each row for each of the other scenarios. As in Figure 6 (and also in Figure A1), for the threshold values that correspond to less than 100,000 routing packets in each scenario, the variation of the average delays is within $1 \mathrm{~ms}$ and that of STDs is within $5 \mathrm{~ms}$. Note that these numbers are the scale units of the delay and STD charts, respectively.
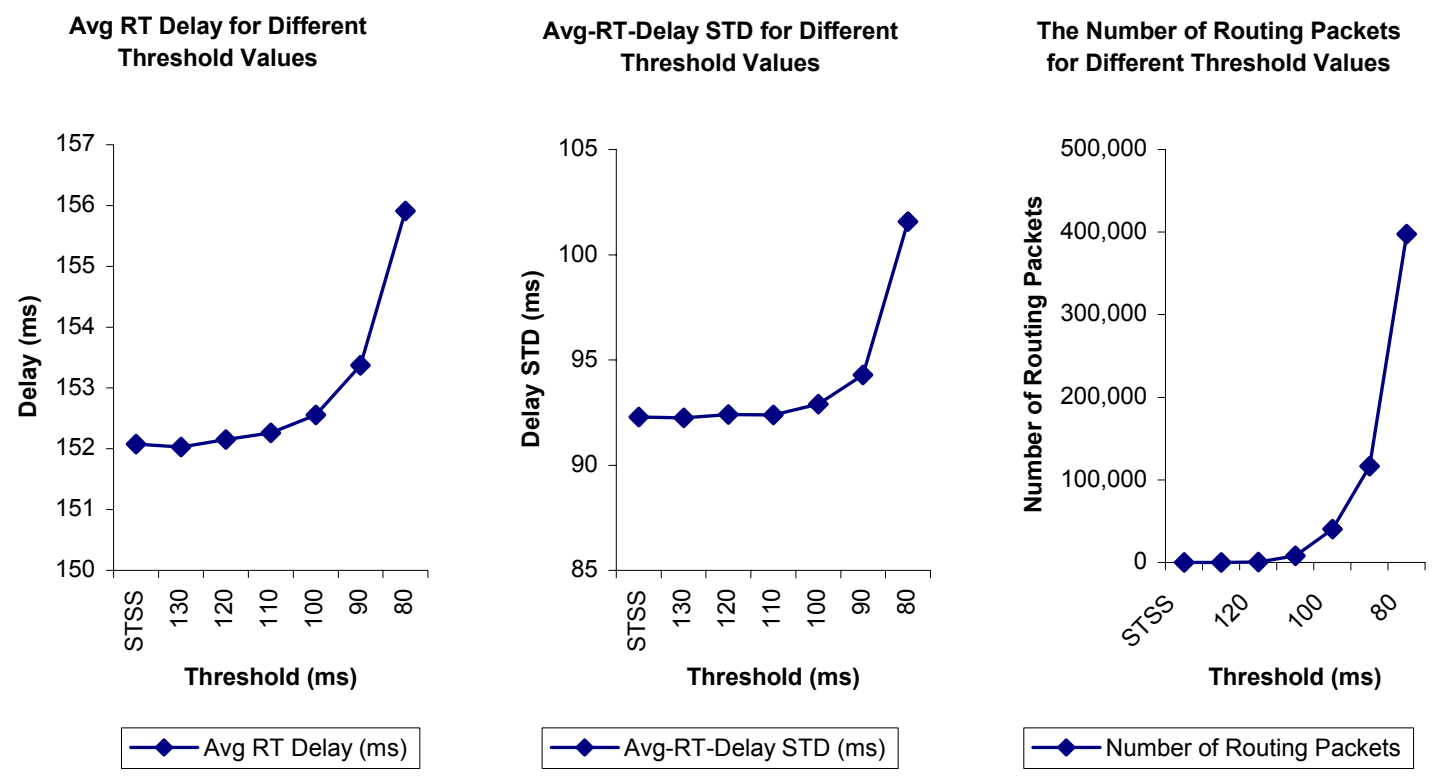

Figure 6 Impact of varying the threshold in Scenario N3
(when using the InfoDyn scheme w/ the best $\alpha$ )

The average delay and STD range from 152.0 to $161.9 \mathrm{~ms}$ and from 89.7 to $109.1 \mathrm{~ms}$, respectively, in the best cases of using the InfoDyn scheme (with the best $\alpha$ ) in all scenarios when routing packets are broadcast. Compared with this best case for each scenario, the InfoDyn STSS case with the same best $\alpha$ leads to increases in the average delay and STD by up to $0.1 \mathrm{~ms}$ and $0.3 \mathrm{~ms}$, respectively. The reason why these increases are small is that the impact of a routing packet on routing quality is transient: the encountered link delay estimated by the receiving node using the delay measurement contained in the packet soon becomes close to the steady-state value. These results indicate that each node may not need to broadcast link-delay measurements when using the InfoDyn scheme. 


\subsubsection{Impact of varying the $\alpha$ value}

There are two possible sources for the routing-quality improvement: use of the long-term steady-state link-delay means and link-delay estimation with the exponential delay-mean change. To see the influence of each of these factors, we first set $\alpha$ to infinity. Then, the linkdelay means are used without any change in route determination. In the InfoDyn STSS case, this Static-Routing case leads to up to $5 \%$ and $18 \%$ increases in the average delay and STD, respectively, in four scenarios and $2 \%$ and $9 \%$ decreases, respectively, in one scenario compared with the best cases of using SPF. These results mean that the use of the link-delay means is not a source of routing-quality improvement in most cases. However, the use of the best $\alpha$ results in 4 to $11 \%$ and 7 to $22 \%$ decreases in the average delay and STD, respectively, in all scenarios compared with these Static-Routing cases. These results indicate that the selection of the $\alpha$ value is crucial for routing-quality improvement.

The best routing quality is achieved with the same $\alpha$ across all scenarios in the case of using the same threshold value or in the InfoDyn STSS case. For example, Figure 7 shows the effects of using different $\alpha$ values in Scenario N3 in the InfoDyn STSS case. The left and right charts indicate the changes in the average delay and STD, respectively, depending on the $\alpha$ value. As in the figure, the average delay and STD increase as the $\alpha$ value used digresses in both directions from the value $(1,000)$ for the best result. Similar trends are observed for the other scenarios. Appendix D (Figure A2 on pages 31 and 32) shows the same two charts in each row for each of the other scenarios. Therefore, if we can find the best or a near-best setting in one case, we may reduce the average delay and STD by using the same setting in other cases. In fact, routing quality is improved for a wide range of $\alpha$ values. Table 4 shows the $\alpha$ ranges of the InfoDyn STSS cases in all scenarios that lead to decreases in the average delay with respect to the best SPF cases. Therefore, the parameter tuning is not required. 
Avg RT Delay for Different

Exponential-Change Rates

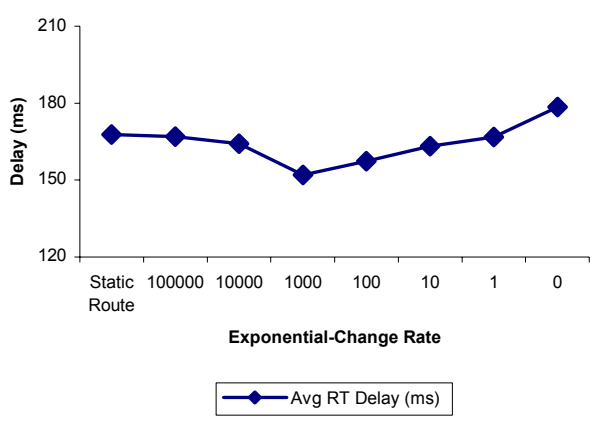

Avg-RT-Delay STD for Different

Exponential-Change Rates

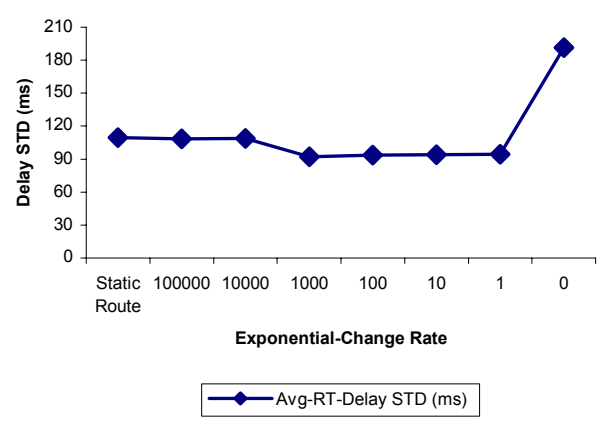

Figure 7 Impact of varying the $\alpha$ value in Scenario N3

(in the InfoDyn STSS case)

Table $4 \alpha$ ranges of the InfoDyn STSS cases leading to decreases in the average RT delay wrt the best SPF cases

\begin{tabular}{|c|c|c|c|c|c|c|c|c|}
\hline \multirow[t]{2}{*}{ Scenario } & \multicolumn{8}{|c|}{ Rate (Circled if Routing Quality is Improved) } \\
\hline & Static Route & 100,000 & 10,000 & 1,000 & 100 & 10 & 1 & 0 \\
\hline No & $\mathrm{O}$ & $\mathrm{O}$ & $\mathrm{O}$ & $\mathrm{O}$ & $\mathrm{O}$ & $\mathrm{O}$ & & \\
\hline N1 & & $\mathrm{O}$ & $\mathrm{O}$ & $\mathrm{O}$ & $\mathrm{O}$ & $\mathrm{O}$ & & \\
\hline N2 & & & & $\mathrm{O}$ & $\mathrm{O}$ & $\mathrm{O}$ & & \\
\hline N3 & & & & $\mathrm{O}$ & $\mathrm{O}$ & & & \\
\hline N4 & & & & $\mathrm{O}$ & $\mathrm{O}$ & & & \\
\hline
\end{tabular}

\subsubsection{Results for different settings of other parameters}

As for InfoDyn routing, each node needs to estimate the steady-state value of each link in order for our approach to be practical. Our simulation results indicate that each node may compute the sample mean of the delay of each local link using standard link-state routing for a long period of time, and flood the sample mean periodically (but, at a lower frequency) so that all other nodes can use it as the steady-state value.

We confirmed via simulation the benefit of broadcasting the sample delay mean for each link at a lower frequency. We changed the implementation of InfoDyn so that the sample mean can be periodically computed and used during the next interval. For the first interval, we used the sample delay mean of the corresponding link computed in a simulation run using SPF with 1 second route-update intervals for the same scenario. In this experiment, we used Scenario N3. Table 5 shows percent decreases in the average round-trip delay per packet, the STD, and the expected number of routing packets of InfoDyn STSS (with the $\alpha$ value set to 1,000) for 
different lengths of sample-mean update interval compared with the best SPF case with 1, 10, and 30 second route-update intervals. Since 1 second interval length leads to the best result in the case of using SPF, the percent change in the routing-packet number is estimated with respect to that for 1 second interval length. As the table shows, if the sample mean is broadcast at 40 second intervals, InfoDyn STSS is better than the best SPF in terms of the average delay, the STD, and the expected number of routing packets used. However, as the frequency increases, the benefit decreases or disappears.

Table 5 Percent decrease in the average delay, the STD, and the expected routingpacket number of InfoDyn STSS compared with the best SPF (w/ 1 second routeupdate interval length) - negative numbers indicate percent increase

\begin{tabular}{c|ccc}
\hline \multirow{3}{*}{$\begin{array}{c}\text { Sample-Mean Broadcast Interval } \\
\text { Length (sec.) of InfoDyn STSS }\end{array}$} & $\begin{array}{c}\text { Average Delay } \\
\text { per Packet }\end{array}$ & STD & $\begin{array}{c}\text { Expected Number of } \\
\text { Routing Packets }\end{array}$ \\
\hline 40 & 3 & 2 & 98 \\
30 & 1 & -6 & 97 \\
20 & -5 & -11 & 95 \\
10 & -9 & -25 & 90 \\
\hline
\end{tabular}

Currently high-speed networks are used in many places. We changed the experimental environment to see whether or not the InfoDyn routing is effective in this situation. We used a high-speed-network setting of $12.5 \mathrm{MB} / \mathrm{s}$ (100 Mbps) link-channel bandwidth, $0.4 \mathrm{~ms}$ datapacket processing time, 5,000 byte data-packet size, and 200 send-window size in Scenario N3 (the all-on-off case). With this setting, the InfoDyn STSS with the best $\alpha$ (which is also 1,000) of the exponential model results in a 2 to $5 \%$ reduction in the average round-trip delay per packet and a $3 \%$ increase to $10 \%$ decrease in the STD compared with the best cases of using SPF with 1, 10, and 30 second route-update intervals. This result demonstrates that the InfoDyn routing can also be effective for high-speed networks. 


\section{Related Work}

Typically, delays vary and change rapidly in a network. For example, at a fine-grained level, the characteristics of the Internet are highly dynamic [Agrawala, 1998]. Such dynamics in networks make it difficult to estimate encountered link delays. Many researchers have investigated the dynamic behavior of networks such as the dynamics of end-to-end Internet packet delays. [Agrawala, 1998; Labowitz, 1998; Paxson, 1999; Pointek, 1997; Sanghi, 1993].

For statistical uncertainty modeling concerning information estimation, there are two basic approaches: modeling based on past observations followed by extrapolation, and modeling via the analysis of factors that determine the information at the target estimation time. An example of the first modeling approach is a time-series model such as an AutoRegressive Integrated Moving Average (ARIMA) model [Box, 1994; Chatfield, 1984]. An example of the second is a regression model for factor(s)-and-effect information pairs (or tuples). The parameters of both modeling approaches can be estimated using least-squares fitting [Trivedi, 1982].

Our research is fundamentally related to understanding the temporal dynamics of information and information systems. Information plays a major role in the operation of systems. In general, such information used in or generated by systems is also dynamic in nature. The Information-Dynamics framework [Agrawala, 2000] provides a new perspective for systems with a focus on information, information usefulness (or "value"), and the changes of information and its usefulness over time. Hence, the framework allows us to better understand the interactions between different components of a system. Such better understanding provides a basis for better system design and implementation. We have performed research on improving link-state routing from an information-dynamics perspective.

Many researchers have addressed time-related issues regarding the model of time and its granularity, time representation, information processing, and distributed computing. Levi and Agrawala [Levi, 1990] recognized the importance of an appropriate representation of time in a variety of applications. Dyreson et al. [Dyreson, 2000] provided a formal model of time and its granularity in a database context. Lamport [Lamport, 1978] defined the "happen before" relationship as a partial ordering of events in distributed systems where entities communicate 
via messages. Based on this relationship, Chandy [Chandy, 1985] developed an algorithm by which an entity can compute a global state of the system.

Regarding information systems, many people have recently performed research on the behavior of entities that exchange information. Kephart et al. [Kephart, 1998] investigated the dynamics of an information-filtering economy. Later, Brooks et al. [Brooks, 2000] showed that a price war in the information goods market can be avoided by taking on different strategies to target a niche. There have also been attempts to model and/or predict e-commerce entities' behavior using a stochastic model [Fader, 2000]. 


\section{Conclusion and Future Work}

Regarding link-state routing, we have studied the issue of estimating and using future link delays. Our simulation results indicate that our approach to solve this problem is promising. To address the future-delay issue, we developed a routing approach based on a new exponential-model-based link-delay-estimation technique, and implemented a routing scheme that uses this approach. When we compared this routing scheme with SPF via simulation for various FTP-workload scenarios with the NSF-T1-backbone network topology, we found that our routing scheme could achieve $100 \%$ reductions in routing traffic with up to $8 \%$ and $22 \%$ decreases of the average round-trip delay per packet and the standard deviation, respectively, in high-load conditions. In comparison studies for the same experimental environment with a high-speed-network setting, we found that our scheme could lead to $100 \%$ reductions in routing traffic with up to $5 \%$ and $10 \%$ decreases of the delay and the standard deviation, respectively, in high-load conditions. These routing-traffic reduction and routing-quality improvements resulted from the estimation of future (encountered) link delays based on the dynamics of the expected link delay given an instantaneous link-delay measurement, and from the consideration of the dynamic usefulness of the link-delay measurement via this estimation.

There will be various issues regarding the practical use of our approach. We plan to provide a guideline for this practical use. In this direction, we will characterize the situations where we can improve link-state routing by using our approach. This characterization study is important for the practical use of our approach because simulation studies suggest that the benefit of using this approach can vary depending on the pattern of the network workload and/or on the characteristics of the network. For this research, we plan to investigate the effectiveness of our approach via extensive simulation studies with different patterns of dynamic workload and/or with different parameter settings for the network. Based on the results of these studies, we will determine the characteristics of the situations that lead to significant routing-traffic reductions with routing-quality enhancements in the case of using our approach, compared with standard link-state routing. 


\section{Appendix}

\section{A. Theorem Proof}

Theorem If $x(t)$ is a stochastic process and has the following additive form for a constant $m$ and a positive constant $\Delta t$ :

$$
x(t+\Delta t)-m=\beta\{x(t)-m\}+(1-\beta) v(t),
$$

then

(i) $E\{x(t)\}=m, \operatorname{Var}\{x(t)\}$ is a constant (denoted by $\sigma^{2}$ ), and the autocorrelation function

$$
\rho\left(t, t_{0}\right)=E\left[\left\{x\left(t_{0}\right)-m\right\}\{x(t)-m\}\right] / \sigma^{2}
$$

$\left(t=t_{0}+k(\Delta t)\right.$ for a non-negative integer $k$ ) decreases exponentially as $t-t_{0}$ (or $k$ ) increases (depending only on the lag), and

(ii) $E\left\{x(t) \mid x\left(t_{0}\right)=x_{0}\right\}=x_{0}+\left(m-x_{0}\right)\left\{1-\rho\left(t, t_{0}\right)\right\}$,

where $\beta$ is a constant such that $0<\beta<1$, and $v(t)$ is an independent white-noise process such that $E\{v(t)\}=0$ and $\operatorname{Var}\{v(t)\}=E\left\{v^{2}(t)\right\}=\sigma_{v}^{2}$ (positive constant) $\square$.

\section{Proof:}

Let us first prove Part (i).

The additive form can be rewritten as

$$
x(t)-m=\beta\{x(t-\Delta t)-m\}+(1-\beta) v(t-\Delta t)
$$

By successive substitution in (1),

$$
\begin{aligned}
& x(t)-m=\beta[\beta\{x(t-2(\Delta t))-m\}+(1-\beta) v(t-2(\Delta t))]+(1-\beta) v(t-\Delta t) \\
& =\beta^{2}[\beta\{x(t-3(\Delta t))-m\}+(1-\beta) v(t-3(\Delta t))] \\
& +(1-\beta) \beta v(t-2(\Delta t))+(1-\beta) v(t-\Delta t) \\
& =\text { : }
\end{aligned}
$$


By using the eventual result of (2),

$$
\begin{aligned}
x(t)-m & =(1-\beta)\left\{v(t-\Delta t)+\beta v(t-2(\Delta t))+\beta^{2} v(t-3(\Delta t))+\cdots\right\} \\
& =(1-\beta) \sum_{i=1}^{\infty}\left\{\beta^{i-1} v(t-i(\Delta t))\right\} \quad \cdots \ldots \ldots \ldots \ldots \ldots \ldots \ldots \ldots \ldots \ldots \ldots \ldots \ldots \ldots \ldots \ldots \ldots \ldots \ldots \ldots \ldots \ldots \ldots
\end{aligned}
$$

Taking the expectation on both sides of (3) yields

$$
E\{x(t)-m\}=(1-\beta) \sum_{i=1}^{\infty}\left[\beta^{i-1} E\{v(t-i(\Delta t))\}\right]
$$

Since $E\{v(t)\}=0$, it follows from (4) that

$$
E\{x(t)-m\}=0
$$

Since $E\{x(t)-m\}=E\{x(t)\}-m$, it follows from (5) that

$$
E\{x(t)\}=m
$$

Taking the expectation on the squares of both sides of (3) yields

$$
\begin{aligned}
E\left[\{x(t)-m\}^{2}\right]= & (1-\beta)^{2} E\left[\left[\sum_{i=1}^{\infty}\left\{\beta^{i-1} v(t-i(\Delta t))\right\}\right]^{2}\right] \\
= & (1-\beta)^{2} \sum_{i=1}^{\infty}\left[\beta^{2(i-1)} E\left\{v^{2}(t-i(\Delta t))\right\}\right] \\
& +(1-\beta)^{2} \sum_{i=1}^{\infty} \sum_{j=1, j \neq i}^{\infty}\left[\beta^{i-1} \beta^{j-1} E\{v(t-i(\Delta t)) v(t-j(\Delta t))\}\right] \ldots
\end{aligned}
$$

Since $E\left\{v\left(t_{1}\right) v\left(t_{2}\right)\right\}=0$ if $t_{1} \neq t_{2}$, and $E\left\{v^{2}(t)\right\}=\sigma_{v}^{2}$, it follows from (7) that

$$
E\left[\{x(t)-m\}^{2}\right]=(1-\beta)^{2} \sigma_{v}^{2} \sum_{i=1}^{\infty} \beta^{2(i-1)}
$$

Since $0<\beta<1$, it follows from (8) that

$$
\begin{aligned}
E\left[\{x(t)-m\}^{2}\right] & =(1-\beta)^{2} \sigma_{v}^{2}\left\{1 /\left(1-\beta^{2}\right)\right\} \\
& =\left\{(1-\beta)^{2} /\left(1-\beta^{2}\right)\right\} \sigma_{v}^{2}
\end{aligned}
$$

Since $\operatorname{Var}\{x(t)\}=E\left[\{x(t)-m\}^{2}\right]$

$$
\operatorname{Var}\{x(t)\}=\left\{(1-\beta)^{2} /\left(1-\beta^{2}\right)\right\} \sigma_{v}^{2}
$$


Since $\sigma^{2}=\operatorname{Var}\{x(t)\}$, and $x(t)-m=(1-\beta) \sum_{i=1}^{\infty}\left\{\beta^{i-1} v(t-i(\Delta t))\right\} \quad$ (3),

$$
\begin{aligned}
\rho\left(t_{0}+(k \Delta t), t_{0}\right) & =(1-\beta)^{2} E\left[\sum_{i=1}^{\infty}\left\{\beta^{i-1} v\left(t_{0}-i(\Delta t)\right)\right\} \sum_{j=1}^{\infty}\left\{\beta^{j-1} v\left(t_{0}+(k \Delta t)-j(\Delta t)\right)\right\}\right] / \sigma^{2} \\
& =(1-\beta)^{2} \sum_{i=1}^{\infty} \sum_{j=1}^{\infty}\left[\beta^{i-1} \beta^{j-1} E\left\{v\left(t_{0}-i(\Delta t)\right) v\left(t_{0}+(k \Delta t)-j(\Delta t)\right)\right\}\right] / \sigma^{2}
\end{aligned}
$$

Since $E\left\{v\left(t_{1}\right) v\left(t_{2}\right)\right\}=0$ if $t_{1} \neq t_{2}, E\left\{v^{2}(t)\right\}=\sigma_{v}^{2}$, and $0<\beta<1$, it follows from (11) that

$$
\begin{aligned}
\rho\left(t_{0}+(k \Delta t), t_{0}\right) & =(1-\beta)^{2} \sum_{i=1}^{\infty}\left\{\beta^{i+k-1} \beta^{i-1} \sigma_{v}^{2}\right\} / \sigma^{2} \\
& =(1-\beta)^{2} \sigma_{v}^{2} \beta^{k} \sum_{i=1}^{\infty} \beta^{2(i-1)} / \sigma^{2} \\
& =(1-\beta)^{2} \sigma_{v}^{2} \beta^{k} /\left\{\left(1-\beta^{2}\right) \sigma^{2}\right\}
\end{aligned}
$$

Since $\sigma^{2}=\left\{(1-\beta)^{2} /\left(1-\beta^{2}\right)\right\} \sigma_{v}^{2}(10)$, it follows from (12) that

$$
\begin{aligned}
\rho\left(t_{0}+(k \Delta t), t_{0}\right) & =\sigma^{2} \beta^{k} / \sigma^{2} \\
& =\beta^{k} \quad \ldots \ldots .
\end{aligned}
$$

Therefore, $E\{x(t)\}=m$ (6), $\operatorname{Var}\{x(t)\}$ is a constant (10), and since $t=t_{0}+k(\Delta t)$ and $0<\beta<1$, it follows from (13) that the autocorrelation function $\rho\left(t, t_{0}\right)$ decreases exponentially as $t-t_{0}$ (or $k$ ) increases (depending only on the lag).

From now, let us prove Part (ii).

For $t_{0}+\Delta t$, it follows from the additive form that

$$
x\left(t_{0}+\Delta t\right)-m=\beta\left\{x\left(t_{0}\right)-m\right\}+(1-\beta) v\left(t_{0}\right)
$$

For $t_{0}+2(\Delta t)=\left(t_{0}+\Delta t\right)+\Delta t$, it follows from the additive form that

$$
x\left(t_{0}+2(\Delta t)\right)-m=\beta\left\{x\left(t_{0}+\Delta t\right)-m\right\}+(1-\beta) v\left(t_{0}+\Delta t\right)
$$

It follows from (14) and (15) that

$$
\begin{aligned}
x\left(t_{0}+2(\Delta t)\right)-m & =\beta\left[\beta\left\{x\left(t_{0}\right)-m\right\}+(1-\beta) v\left(t_{0}\right)\right]+(1-\beta) v\left(t_{0}+\Delta t\right) \\
& =\beta^{2}\left\{x\left(t_{0}\right)-m\right\}+(1-\beta)\left\{\beta v\left(t_{0}\right)+v\left(t_{0}+\Delta t\right)\right\} \quad \ldots \ldots
\end{aligned}
$$

For $t_{0}+3(\Delta t)=\left(t_{0}+2(\Delta t)\right)+\Delta t$, it follows from the additive form that

$$
x\left(t_{0}+3(\Delta t)\right)-m=\beta\left\{x\left(t_{0}+2(\Delta t)\right)-m\right\}+(1-\beta) v\left(t_{0}+2(\Delta t)\right)
$$


It follows from (16) and (17) that

$$
\begin{aligned}
x\left(t_{0}+3(\Delta t)\right)-m= & \beta\left[\beta^{2}\left\{x\left(t_{0}\right)-m\right\}+(1-\beta)\left\{\beta v\left(t_{0}\right)+v\left(t_{0}+\Delta t\right)\right\}\right] \\
& +(1-\beta) v\left(t_{0}+2(\Delta t)\right) \\
= & \beta^{3}\left\{x\left(t_{0}\right)-m\right\}+(1-\beta)\left\{\beta^{2} v\left(t_{0}\right)+\beta v\left(t_{0}+\Delta t\right)+v\left(t_{0}+2(\Delta t)\right\}\right.
\end{aligned}
$$

By using the successive substitution shown in (14), (16), and (18), for $t_{0}+k(\Delta t)(k>0)$,

$$
x\left(t_{0}+k(\Delta t)\right)-m=\beta^{k}\left\{x\left(t_{0}\right)-m\right\}+(1-\beta) \sum_{i=0}^{k-1}\left\{\beta^{k-i-1} v\left(t_{0}+i(\Delta t)\right\}\right.
$$

Since $t=t_{0}+k(\Delta t)$, it follows from (19) that

$$
x(t)-m=\beta^{k}\left\{x\left(t_{0}\right)-m\right\}+(1-\beta) \sum_{i=0}^{k-1}\left\{\beta^{k-i-1} v\left(t_{0}+i(\Delta t)\right\}\right.
$$

(20) can be rewritten as

$$
x(t)=m+\beta^{k}\left\{x\left(t_{0}\right)-m\right\}+(1-\beta) \sum_{i=0}^{k-1}\left\{\beta^{k-i-1} v\left(t_{0}+i(\Delta t)\right\}\right.
$$

The non-white-noise term of the right side of (21) is

$$
m+\beta^{k}\left\{x\left(t_{0}\right)-m\right\}
$$

Given $x\left(t_{0}\right)=x_{0},(22)$ is not a random variable. Then, $x(t)$ gets its randomness only from $(1-\beta) \sum_{i=0}^{k-1}\left\{\beta^{k-i-1} v\left(t_{0}+i(\Delta t)\right\}\right.$. Taking the conditional expectation on both sides of (21) yields

$$
E\left\{x(t) \mid x\left(t_{0}\right)=x_{0}\right\}=m+\beta^{k}\left(x_{0}-m\right)+(1-\beta) \sum_{i=0}^{k-1}\left[\beta^{k-i-1} E\left\{v\left(t_{0}+i(\Delta t) \mid x\left(t_{0}\right)=x_{0}\right\}\right]\right.
$$

Since $x(t)$ can be expressed only with $v(t)$ (3), and $v(t)$ is an independent process,

$$
E\left\{v(t) \mid x\left(t_{0}\right)\right\}=E\{v(t)\}
$$

Since $E\{v(t)\}=0$, it follows from (23) and (24) that

$$
E\left\{x(t) \mid x\left(t_{0}\right)=x_{0}\right\}=m+\beta^{k}\left(x_{0}-m\right)
$$

As this non-random term keeps changing its value (when $x_{0} \neq m$ ), $E\left\{x(t) \mid x\left(t_{0}\right)=x_{0}\right\} \neq m$. Since $t=t_{0}+k(\Delta t)$ and $\rho\left(t_{0}+k(\Delta t), t_{0}\right)=\beta^{k}$ (13), it follows from (25) that

$$
E\left\{x(t) \mid x\left(t_{0}\right)=x_{0}\right\}=m+\rho\left(t, t_{0}\right)\left(x_{0}-m\right)
$$

Since $m+\rho\left(t, t_{0}\right)\left(x_{0}-m\right)=x_{0}+\left(m-x_{0}\right)\left\{1-\rho\left(t, t_{0}\right)\right\}$, it follows from (26) that

$$
E\left\{x(t) \mid x\left(t_{0}\right)=x_{0}\right\}=x_{0}+\left(m-x_{0}\right)\left\{1-\rho\left(t, t_{0}\right)\right\}
$$




\section{B. Additional Theorem and Proof}

Additional Theorem If $x(t)$ is a stochastic process and has the following additive form for a constant $m$ and a positive constant $\Delta t$ :

$$
x(t+\Delta t)-m=\beta\{x(t)-m\}+(1-\beta) v(t),
$$

then $\sigma^{2}\left(x_{0}, t_{0}, t\right) \quad\left(t=t_{0}+k(\Delta t)\right.$ for a non-negative integer $\left.k\right)$ decays exponentially over time to $\sigma^{2}$, where $\sigma^{2}\left(x_{0}, t_{0}, t\right)$ denotes $\operatorname{Var}\left\{x(t) \mid x\left(t_{0}\right)=x_{0}\right\}, \sigma^{2}$ denotes $\operatorname{Var}\{x(t)\}, \beta$ is a constant such that $0<\beta<1$, and $v(t)$ is an independent white-noise process such that $E\{v(t)\}=0$ and $\operatorname{Var}\{v(t)\}=E\left\{v^{2}(t)\right\}=\sigma_{v}^{2}$ (positive constant) $\square$.

\section{Proof:}

The equation (21) in the proof of Theorem (page 26) is

$$
x(t)=m+\beta^{k}\left\{x\left(t_{0}\right)-m\right\}+(1-\beta) \sum_{i=0}^{k-1}\left\{\beta^{k-i-1} v\left(t_{0}+i(\Delta t)\right\}\right.
$$

Since $\sigma^{2}\left(x_{0}, t_{0}, t\right)=\operatorname{Var}\left\{x(t) \mid x\left(t_{0}\right)=x_{0}\right\}$, it follows from (1) that

$$
\begin{aligned}
\sigma^{2}\left(x_{0}, t_{0}, t\right)= & \operatorname{Var}\left[(1-\beta) \sum_{i=0}^{k-1}\left\{\beta^{k-i-1} v\left(t_{0}+i(\Delta t)\right\} \mid x\left(t_{0}\right)=x_{0}\right]\right. \\
= & (1-\beta)^{2} E\left[\left[\sum_{i=0}^{k-1}\left\{\beta^{k-i-1} v\left(t_{0}+i(\Delta t)\right\}\right]^{2} \mid x\left(t_{0}\right)=x_{0}\right]\right. \\
& -(1-\beta)^{2} E^{2}\left[\sum_{i=0}^{k-1}\left\{\beta^{k-i-1} v\left(t_{0}+i(\Delta t)\right\} \mid x\left(t_{0}\right)=x_{0}\right]\right.
\end{aligned}
$$

Since $x(t)$ can be expressed only with $v(t)$ (3) in the proof of Theorem (page 24), and $v(t)$ is an independent process,

$$
E\left\{v^{2}(t) \mid x\left(t_{0}\right)\right\}=E\left\{v^{2}(t)\right\}
$$


Since $E\left\{v(t) \mid x\left(t_{0}\right)\right\}=E\{v(t)\}$ (24) in the proof of Theorem (page 26), it follows from (2) and (3) that

$$
\begin{aligned}
\sigma^{2}\left(x_{0}, t_{0}, t\right)= & (1-\beta)^{2} E\left[\left[\sum_{i=0}^{k-1}\left\{\beta^{k-i-1} v\left(t_{0}+i(\Delta t)\right\}\right]^{2}\right]\right. \\
& -(1-\beta)^{2} E^{2}\left[\sum_{i=0}^{k-1}\left\{\beta^{k-i-1} v\left(t_{0}+i(\Delta t)\right\}\right]\right.
\end{aligned}
$$

Since $E\left\{v\left(t_{1}\right) v\left(t_{2}\right)\right\}=0$ if $t_{1} \neq t_{2}, E\left\{v^{2}(t)\right\}=\sigma_{v}^{2}$, and $E\{v(t)\}=0$, it follows from (4) that

$$
\begin{aligned}
\sigma^{2}\left(x_{0}, t_{0}, t\right) & =(1-\beta)^{2} \sigma_{v}^{2} \beta^{2 k} \sum_{i=0}^{k-1} \beta^{-2(i+1)} \\
& =(1-\beta)^{2} \sigma_{v}^{2} \beta^{2 k}\left\{\beta^{-2}\left(1-\beta^{-2 k}\right) /\left(1-\beta^{-2}\right)\right\} \\
& =\left[\left\{(1-\beta)^{2} \sigma_{v}^{2}\right\} /\left(\beta^{2}-1\right)\right]\left(\beta^{2 k}-1\right) \quad \ldots \ldots . . .
\end{aligned}
$$

Since $\sigma^{2}=\left\{(1-\beta)^{2} /\left(1-\beta^{2}\right)\right\} \sigma_{v}^{2}$ (10) in the proof of Theorem (page 24), it follows from (5) that

$$
\sigma^{2}\left(x_{0}, t_{0}, t\right)=\sigma^{2}\left(1-\beta^{2 k}\right)
$$

Since $t=t_{0}+k(\Delta t)$ and $0<\beta<1$, it follows from (6) that $\sigma^{2}\left(x_{0}, t_{0}, t\right)$ decays exponentially over time to $\sigma^{2}$. 
C. Figure for the Impact of Varying the Threshold in the Other Scenarios

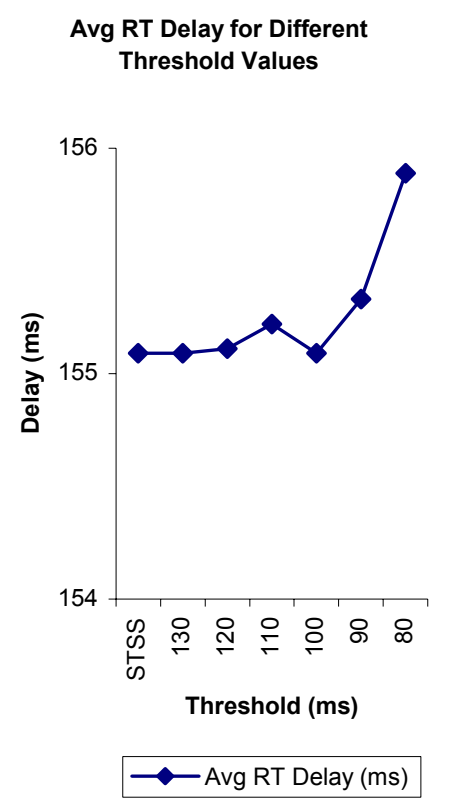

[a]
Avg-RT-Delay STD for Different Threshold Values
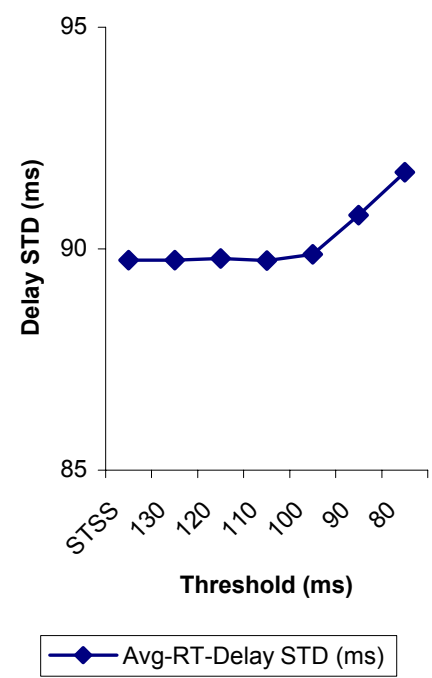

[b]
The Number of Routing Packets for Different Threshold Values

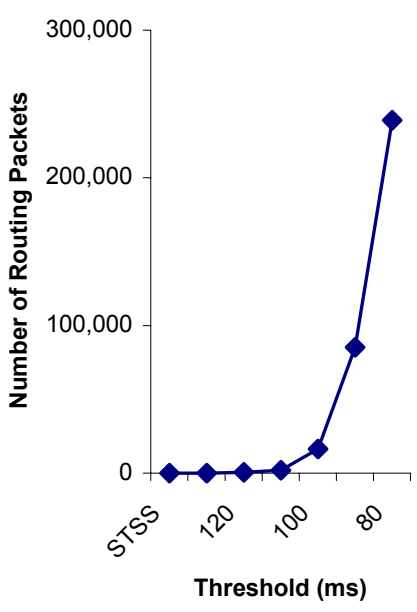

$\neg$ Number of Routing Packets

[c]

([a], [b], and [c]: Scenario N0)

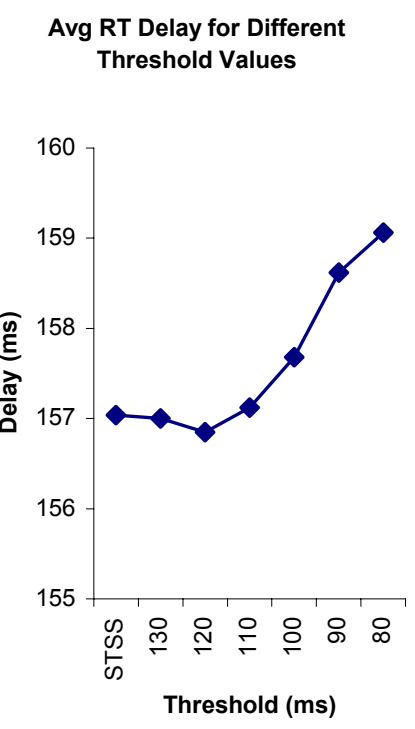

$\sim$ Avg RT Delay (ms)

\section{Avg-RT-Delay STD for Different \\ Threshold Values}

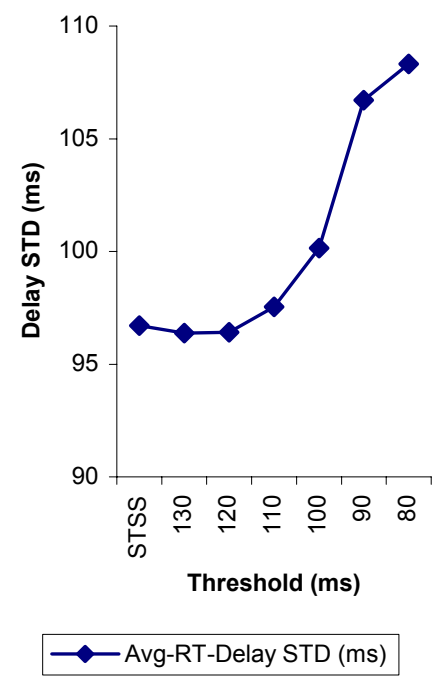

[e]
The Number of Routing Packets for Different Threshold Values

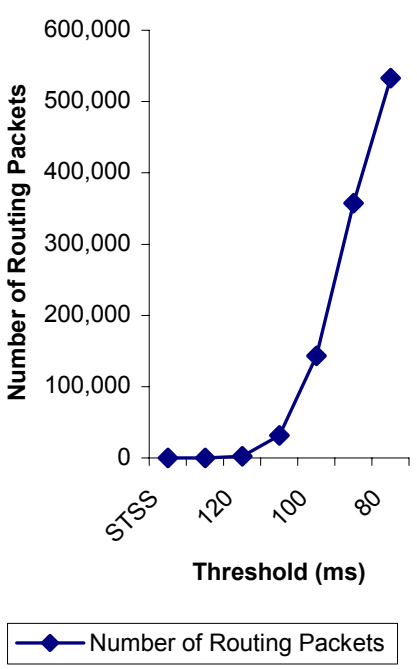

[d]

([d], [e], and [f]: Scenario N1) 


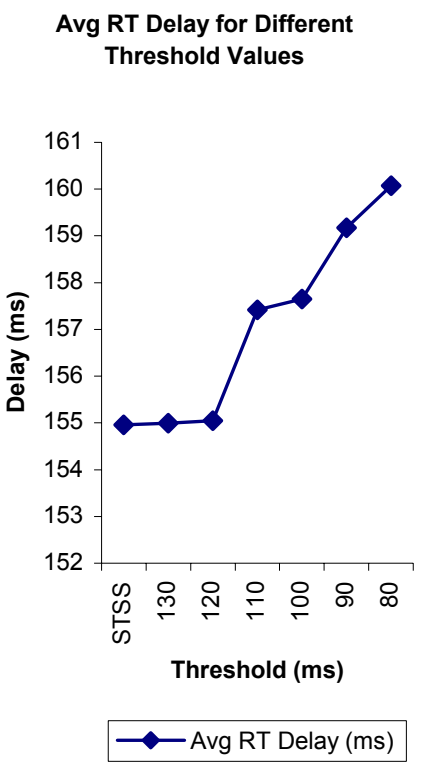

$[g]$
Avg-RT-Delay STD for Different Threshold Values

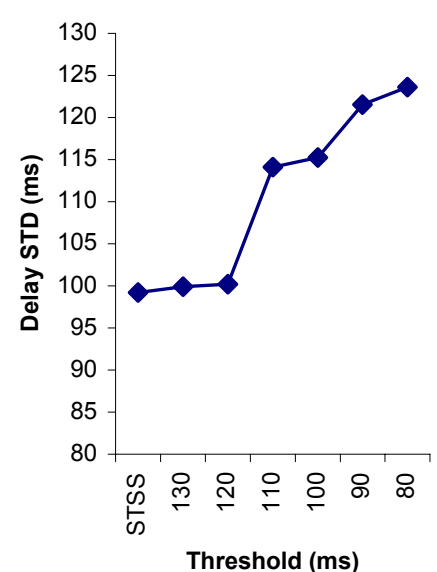

$\multimap$ Avg-RT-Delay STD (ms)

[h]
The Number of Routing Packets for Different Threshold Values

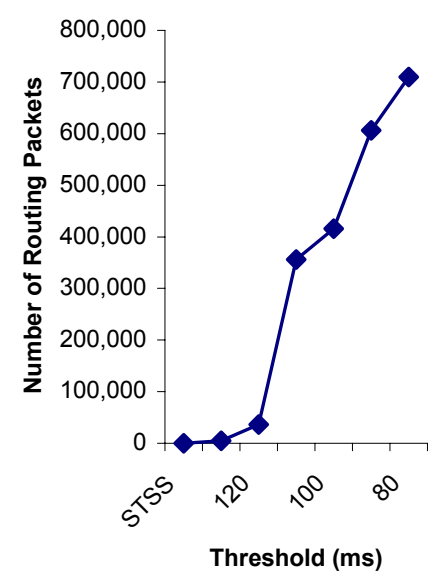

$\neg$ Number of Routing Packets

\section{([g], [h], and [i]: Scenario N2)}

Avg RT Delay for Different Threshold Values

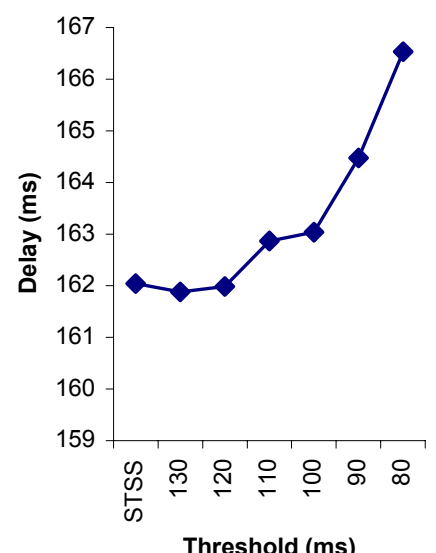

$\multimap$ Avg RT Delay (ms)

[j]
Avg-RT-Delay STD for Different Threshold Values
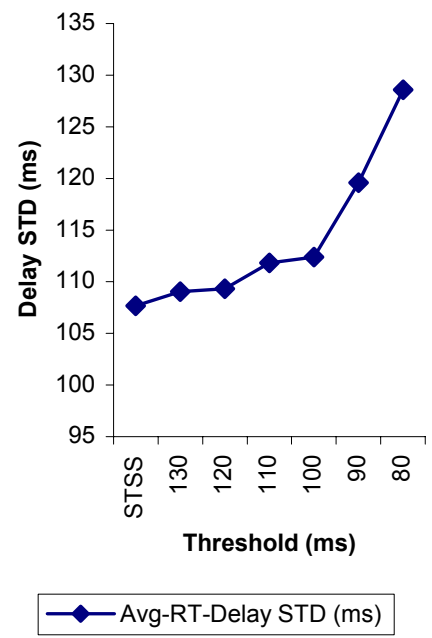

The Number of Routing Packets for Different Threshold Values

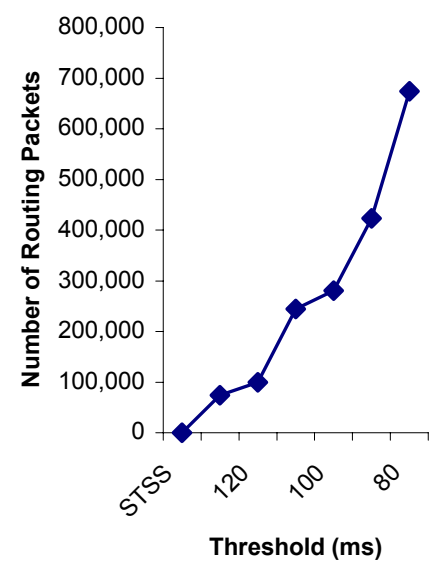

Number of Routing Packets

\section{([j], [k], and [1]: Scenario N4)}

Figure A1 Impact of varying the threshold in Scenarios N0, N1, N2, and N4 (when using the InfoDyn scheme w/ the best $\alpha$ ) 
D. Impact of varying the $\alpha$ value in the Other Scenarios

Avg RT Delay for Different

Exponential-Change Rates

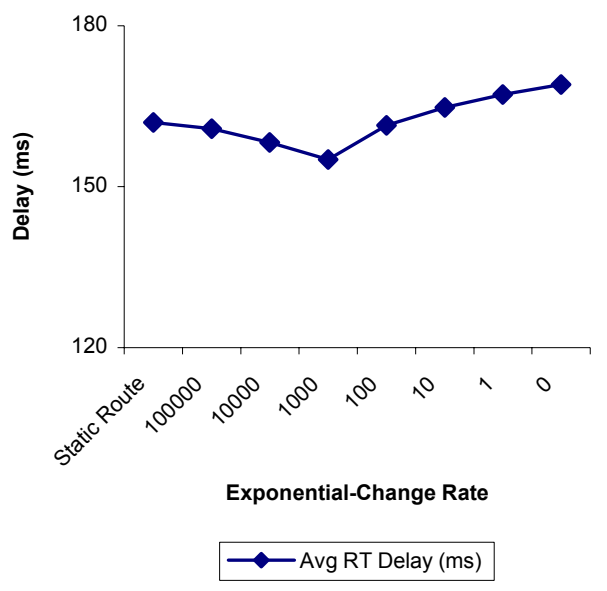

[a]
Avg-RT-Delay STD for Different

Exponential-Change Rates

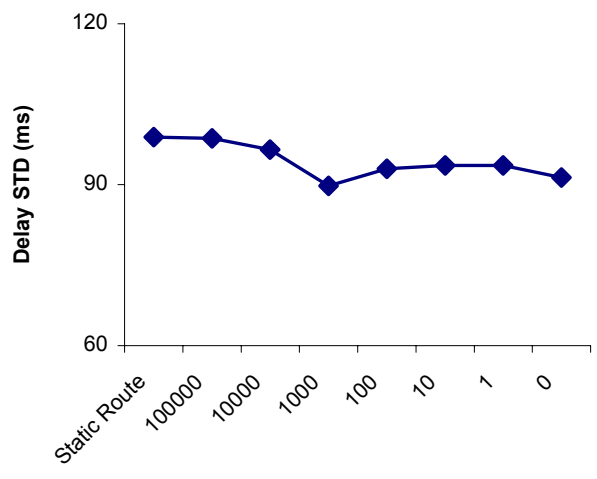

Exponential-Change Rate

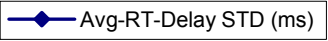

[b]

([a] and [b]: Scenario N0)

Avg RT Delay for Different

Exponential-Change Rates

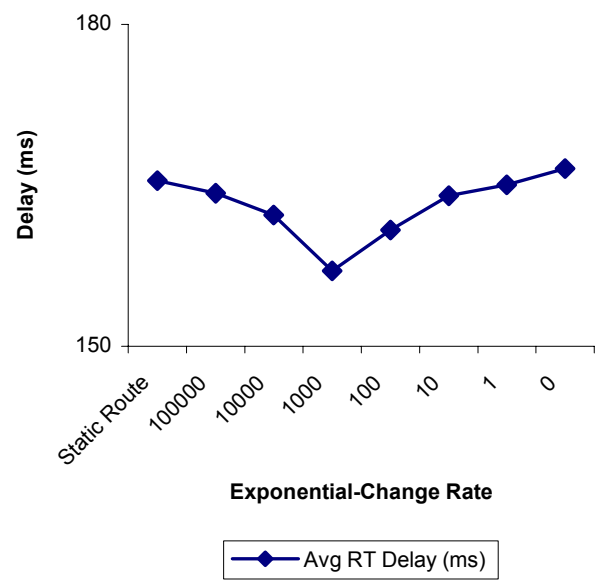

[c]

\begin{abstract}
Avg-RT-Delay STD for Different Exponential-Change Rates
\end{abstract}

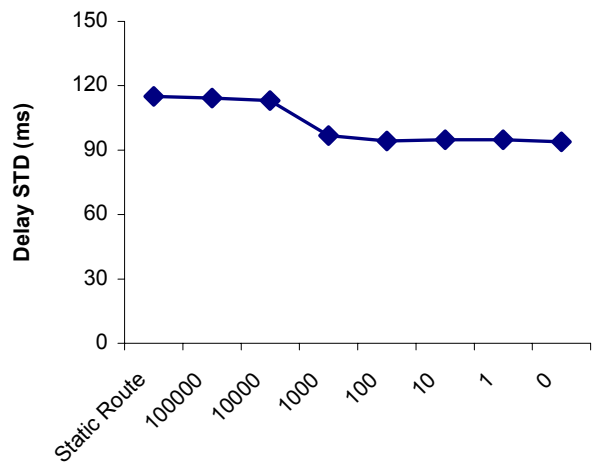

Exponential-Change Rate

Avg-RT-Delay STD (ms)

[d]

([c] and [d]: Scenario N1) 
Avg RT Delay for Different

Exponential-Change Rates

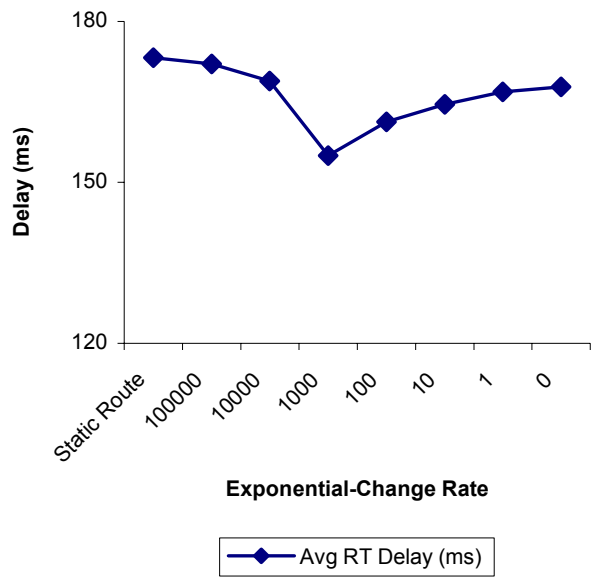

[e]
Avg-RT-Delay STD for Different

Exponential-Change Rates

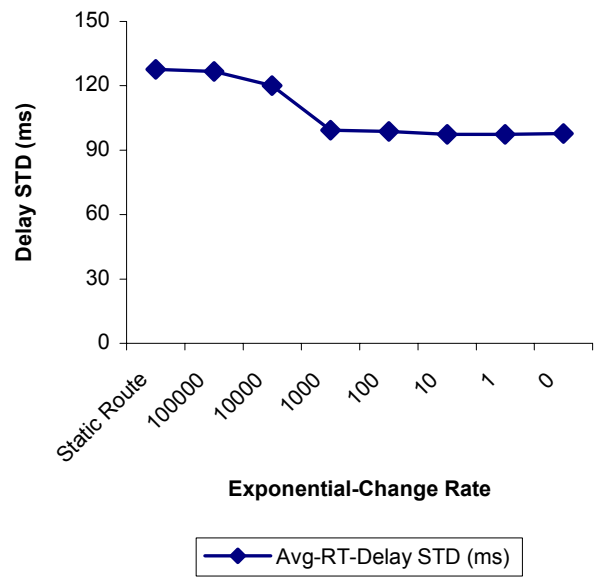

[f]

\section{([e] and [f]: Scenario N2)}

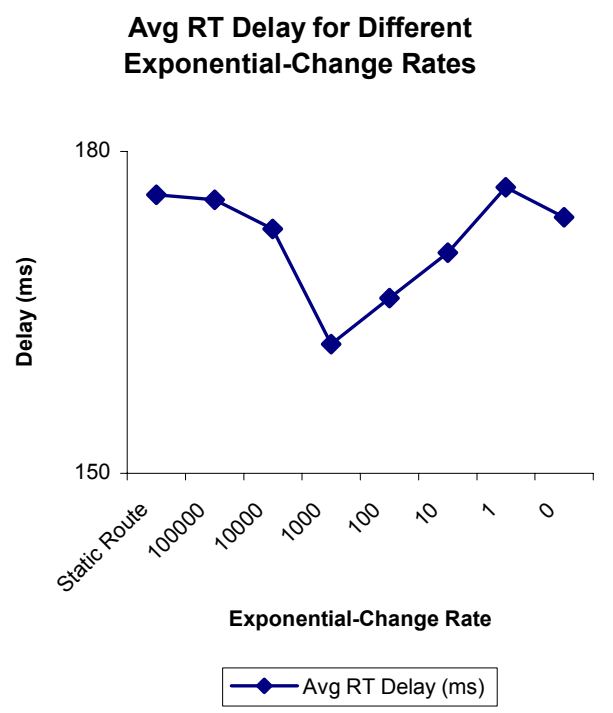

$[g]$

\section{Avg-RT-Delay STD for Different Exponential-Change Rates}

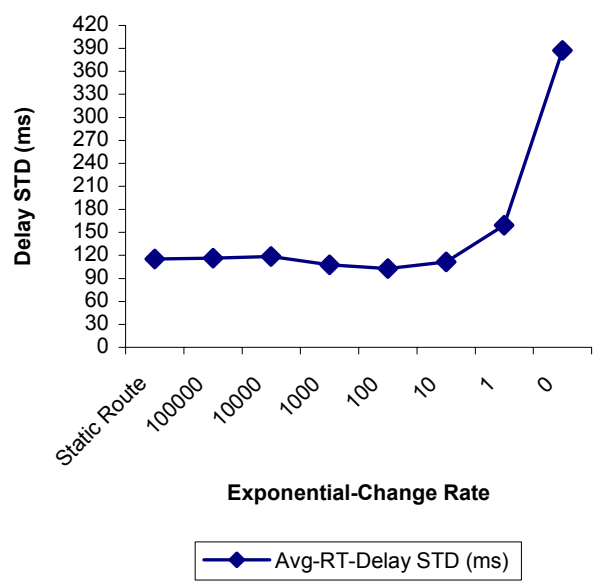

[h]

([g] and [h]: Scenario N4)

Figure A2 Impact of varying the $\alpha$ value in Scenarios N0, N1, N2, and N4 (In the InfoDyn STSS cases) 
December 12, 2002

\section{Bibliography}

[Agrawala, 2000] A.K. Agrawala, R. Larsen, and D. Szajda "Information Dynamics: An InformationCentric Approach to System Design," Proceedings of the International Conference on Virtual Worlds and Simulation, January 2000.

[Agrawala, 1998] A.K. Agrawala, The NetCalliper Project, http://www.cs.umd.edu/projects/sdag/netcalliper, 1998.

[Alaettinoglu, 1994] C. Alaettinoglu, A.U. Shankar, K. Dussa-Zieger, and I. Matta, "Design and Implementation of MaRS: A Routing Testbed," Journal of Internetworking: Research \& Experience, 5 (1):17-41, 1994.

[Box, 1994] G.E.P. Box, G.M. Jenkins, and G.C. Reinsel, Time Series Analysis: Forecasting and Control, Prentice Hall, 1994.

[Brooks, 2000] C.H. Brooks, E.H. Durfee, and R. Das, "Price Wars and Niche Discovery in an Information Economy," Proceedings of the Second ACM Conference on Electronic Commerce, Minneapolis, Minnesota, October 2000, pp. 95-106.

[Chandy, 1985] K.M. Chandy and L. Lamport, "Distributed Snapshots: Determining Global States of Distributed Systems," ACM Trans. Comp. Syst., 3(1), pp. 63-75, 1985.

[Chatfield, 1984] C. Chatfield, The Analysis of Time Series: an Introduction, Chapman and Hall, London \& New York, 1984.

[Dijkstra, 1959] E.W. Dijkstra, "A Note on Two Problems in Connection with Graphs," Numerische Mathematik, 1, pp. 269-271, 1959.

[Dyreson, 2000] C.E. Dyreson, W.S. Evans, H. Lin, and R.T. Snodgrass, "Efficiently Supporting Temporal Granularities," IEEE Trans. Knowledge and Data Eng., 12 (4), pp. 568-586, 2000.

[Fader, 2000] P.S. Fader and B.G.S. Hardie, "Forecasting Repeat Sales at CDNOW: A Case Study," Working Paper, The Wharton School of the University of Pennsylvania, 2000.

[Glazer, 1990] D.W. Glazer and C. Tropper, "A New Metric for Adaptive Routing in the Chaotic and Unbalanced Traffic Environment,” IEEE Trans. Comm., 38(3), pp. 360-367, 1990.

[Kaur, 2000] H.T. Kaur and K.S. Vastola, "The Tunability of Network Routing Using Online Simulation," Proceedings of Symposium on Performance Evaluation of Computer and Telecommunication Systems (SPECTS), Vancouver, B.C., Canada, July 2000.

[Kephart, 1998] J.O. Kephart, "Dynamics of an Information-Filtering Economy," Proceedings of the Second International Workshop on Cooperative Information Agents (CLA), Paris, July 1998, pp. 160-171.

[Khanna, 1989] A. Khanna and J. Zinky, "The Revised ARPANET Routing Metric," Proceedings of ACM SIGCOMM, pp. 45-56, 1989.

[Labowitz, 1998] C. Labowitz, G.Malan, and F. Jahanian, “Internet Routing Instability,” IEEE/ACM Trans. Networking, 6 (5):515-528, October 1998.

[Lamport, 1978] L. Lamport, "Time, Clocks, and the Ordering of Events in a Distributed Systems," Communications of the ACM, 21(7), pp. 558-565, 1978.

[Levi, 1990] S.T. Levi and A.K. Agrawala, Real Time System Design, McGraw-Hill Publishing Company, 1990.

[Paxson, 1999] V. Paxson, "End-to-End Internet Packet Dynamics," IEEE/ACM Trans. Networking, 7 (3):277-292, June 1999.

[Peterson, 1996] LL.Peterson and B.S. Davie, Computer Network: A Systems Approach, Reprographic Services, 1996.

[Pointek, 1997] J. Pointek, F. Shull, R. Tesoriero and A.K. Agrawala, "NetDyn Revisited: A Replicated Study of Network Dynamics," Computer Networks and ISDN Systems, 29(7):831-840, August 1997.

[Rosen, 1980] E.C. Rosen, "The Updating Protocol of ARPANET's New Routing Algorithm," Computer Networks, 4(1), pp. 11-19, 1980.

[Sanghi, 1993] D. Sanghi, O. Gudmundsson, and A.K. Agrawala, "A Study of Network Dynamics," Computer Networks and ISDN Systems, 26(3), pp. 371-378, November 1993. 
[Shankar, 1992] A.U. Shankar, C. Alaettinoglu, I. Matta, and K. Dussa-Zieger, "Performance Comparison of Routing Protocols using MaRS: Distance-Vector versus Link-State," Proceedings of ACM SIGMETRICS, pp. 181-192, 1992.

[Trivedi, 1982] K.S. Trivedi, Probability \& Statistics with Probability, Quening and Computer Science Applications, Prentice Hall, 1982. 